\title{
Estrategias de resolución de problemas y creencias de estudiantes universitarios (estudio de casos)
}

DOI: https://doi.org/10.33262/ap.v1i3.5

\begin{abstract}
(c) (1) ()(2)
University students 'problem and belief resolution strategies (case studies)
\end{abstract}

Armando A. Fonte Águila. ${ }^{1} \&$ Mario Varela Nualles. ${ }^{2}$

\begin{abstract}
.
The knowledge of the strategies and beliefs that the students bring from the previous teaching levels, would allow the teachers in Mathematics to readjust the methodological treatment of the contents and give differentiated attention.

This research proposes a scientific methodology based on the methodological conception of Case Studies to isolate and describe the strategies used by first-year students of the Bachelor of Accounting and Finance, Bachelor of Agronomy and Agricultural Engineering, of the Agrarian University of Havana; as well as the first-year students of the Bachelor's degree in Pedagogy, specialties of Physics and Computer Science of the Polytechnic School of Moxico, Republic of Angola. It also seeks to isolate some of the beliefs that hold back the development of successful solution processes.

As results, examples of the strategies and beliefs manifested are exposed and they are derived through descriptive statistics, particularities by careers and individual diagnoses by elements of knowledge.
\end{abstract}

Keywords: Problem solving, strategies, beliefs, reflective, thoughtless, routine and non-routine problem.

\footnotetext{
${ }^{1}$ Universidad Agraria de La Habana "Fructuoso Rodríguez Pérez". La Habana, Cuba.

${ }^{2}$ Instituto Nacional de Ciencias Agrícolas. La Habana, Cuba.
} 


\section{Resumen.}

El conocimiento de las estrategias y creencias que traen los estudiantes de los niveles de enseñanza precedentes, permitiría a los docentes en Matemáticas readecuar el tratamiento metodológico de los contenidos y dar atención diferenciada.

Esta investigación propone una metodología científica basada en la concepción metodológica de los Estudios de Caso para, mediante diagnóstico, aislar y describir las estrategias que utilizan los estudiantes de primer año de las carreras Licenciatura en Contabilidad y Finanzas, Licenciatura en Agronomía e Ingeniería Agrícola, de la Universidad Agraria de La Habana; así como de los estudiantes de primer año de la carrera Licenciatura en Pedagogía, especialidades de Física e Informática de la Escuela Superior Politécnica de Moxico, República de Angola. Se persigue también aislar algunas de las creencias que frenan el desarrollo de procesos exitosos de solución.

Como resultados se exponen ejemplos de las estrategias y creencias manifestadas y se derivan mediante estadísticas descriptivas particularidades por carreras y los diagnósticos individuales por elementos del conocimiento.

Palabras claves: Resolución de problemas, estrategias, creencias, reflexivo, irreflexivo, problema rutinario y no rutinario.

\section{Introducción.}

Autores plantean que en las aulas no se enseña a resolver problemas (Campistrous y Rizo, 1999). Ante situaciones problémicas, los estudiantes utilizan estrategias rutinarias y adquieren un sistema de creencias negativas para el desarrollo de procesos exitosos de solución, determinando el carácter irreflexivo de las estrategias que utilizan. Fonte (2003)

En esta investigación, se abordan las estrategias y creencias que mantienen los estudiantes que comienzan estudios superiores en la Universidad Agraria de La Habana (UNAH), en las carreras de Licenciatura en Contabilidad y Finanzas, Licenciatura en Agronomía e Ingeniería Agrícola. Para llevarla a cabo se ha trazado el siguiente Diseño de investigación.

Problema científico: ¿Cuáles son las estrategias que los estudiantes del primer año de las carreras Licenciatura en Contabilidad y Finanzas, Licenciatura en Agronomía e Ingeniería Agrícola de la UNAH y los de primer año de la carrera Licenciatura en Pedagogía, especialidades de Física e Informática de la Escuela Superior Politécnica de Moxico, Angola, ¿utilizan para resolver problemas y qué creencias limitan esta actividad?

Objeto de la investigación: el proceso de solución de problemas por parte de los estudiantes del estudio. 
Objetivo general: Aislar, mediante un estudio de casos, las estrategias que utilizan los estudiantes para resolver problemas, determinando, si existen, las creencias que limitan las posibilidades de éxito en esta actividad.

Campo de acción: las estrategias que utilizan los estudiantes para resolver problemas y las creencias que se forman en dicho proceso.

\section{Preguntas científicas:}

1. ¿Cuáles son las tendencias actuales de la resolución de problemas y en particular, acerca del concepto de problema?

2. ¿Cuáles son las estrategias propias que utilizan los estudiantes del estudio para la resolución de problemas y cómo determinarlas?

3. ¿Se han formado creencias en los estudiantes acerca de la actividad de resolver problemas? ¿Cuáles son?

\section{Métodos:}

\section{A nivel teórico:}

Análisis y síntesis e Inducción y Deducción, para determinar estrategias y creencias.

Histórico Lógico: Para el estudio de las fuentes de información y el procesamiento de los fundamentos científicos.

\section{A nivel empírico:}

Test y entrevistas: Para la selección de los problemas y el reconocimiento de las intensiones de los estudiantes en sus respuestas.

Estadísticas descriptivas: en la confección de tablas de conteo.

Novedad científica: consiste en la realización, por primera vez en la enseñanza superior, del estudio de las estrategias y creencias de los estudiantes en resolución de problemas

Aporte y significación práctica: Se completa en Cuba el ciclo de los estudios de este tipo en todos los niveles de enseñanza y se extiende el estudio a la Escuela Superior Politécnica de Moxico.

\section{Desarrollo.}

\section{Basamento teórico.}

La tendencia que toma como basamento científico esta investigación es la que se reconoce como la Enseñanza de la Resolución de Problemas, la cual sostiene que “... el pensamiento 
es una actividad que tiene lugar fundamentalmente cuando el hombre resuelve problemas" Labarrere (1998), y que se debe organizar la enseñanza de la Matemática enseñando a resolver problemas.

Se asumen con Campistrous y Rizo (1999) los conceptos siguientes:

Concepto de problema: "Un problema es toda situación en la que hay un planteamiento inicial y una exigencia que obliga a transformarlo. La vía para pasar de la situación inicial a la nueva situación exigida, tiene que ser desconocida: cuando es conocida deja de ser un problema. El individuo quiere hacer la transformación, es decir, quiere resolver el problema"

Un procedimiento es rutinario y por extensión, un problema es rutinario, si, “... en el proceso de solución se pueden encontrar las vías de solución de una manera directa en el propio contenido de la asignatura que se aborda en la escuela, y en ellos se emplean procedimientos que no llegan a ser propiamente algorítmicos, pero tampoco llegan a ser procedimientos heurísticos de búsqueda abierta, sino de una determinación o selección entre dos o más rutinas ya preestablecidas que sí son, por lo general, procedimientos algorítmicos",

Concepto de estrategia de solución de problemas: "Una estrategia es un conjunto de acciones o decisiones que en determinado orden realiza un alumno para obtener la respuesta a un problema con un mínimo de esfuerzo previendo contra resultados no esperados" (Fonte, 2003).

Una estrategia es irreflexiva, cuando responde a un proceder prácticamente automatizado, sin que pase por una etapa de análisis previo u orientación en el problema. En estos casos se asocia la vía de solución a factores puramente externos. En el caso contrario, o sea, cuando para su uso se requiere necesariamente un proceso de análisis previo que permite asociar la vía de solución a factores estructurales y no a factores puramente externos, la hemos denominado estrategias reflexivas" (Campistrous y Rizo, 1999).

\section{Concepción metodológica del estudio realizado.}

\section{Consideraciones acerca de la metodología el Estudio de Casos.}

Son considerados como exámenes intensivos y completos de ejemplos en acción siendo sus técnicas y procedimientos la observación, la entrevista, las grabaciones audiovisuales, notas de campo, etc. (Marcelo y otros, 1991).

\section{Descripción del caso objeto de estudio.}

El diagnóstico se realiza a 30 estudiantes de Licenciatura en Contabilidad y Finanzas, 26 estudiantes de Licenciatura en Agronomía y a 41 estudiantes de Ingeniería agrícola; todos de los primeros años de estas carreras en la UNAH. 
Al total de los estudiantes les fue aplicada la metodología que proponen Campistrous y Rizo (1999), que consta de las acciones siguientes:

I) Aplicación de test escritos (selección de problemas) para reconocer estrategias ya aisladas y clasificadas en estudios anteriores,

II) Análisis de los resultados para establecer hipótesis de las posibles estrategias utilizadas en las soluciones,

III) Entrevistas para confirmar o rechazar las hipótesis antes planteadas,

IV) Análisis exhaustivo para arribar a conclusiones definitivas.

El test se compone de 10 problemas no rutinarios (ver Anexo No. 1).

En Licenciatura en Contabilidad y Finanzas se aplica a cada estudiante cinco de los diez problemas en dos temarios y en las otras dos carreras un solo temario de diez problemas a 26 y 41 estudiantes de la UNAH respectivamente y a 48 estudiantes de la ESPM, tal y como se muestra en la tabla siguiente:

Tabla 1. Resultados del test

\begin{tabular}{ccc}
\hline Carreras & $\begin{array}{c}\text { Total, de } \\
\text { estudiantes }\end{array}$ & $\begin{array}{c}\text { No. de problemas resueltos } \\
\text { por estudiantes }\end{array}$ \\
\hline Lic. en Contabilidad y & 30 & 5 \\
Finanzas (UNAH) & 26 & 10 \\
Lic. en Agronomía (UNAH) & 41 & 10 \\
Ing. Agrícola (UNAH) & 48 & 10 \\
Licenciatura en Pedagogía & 145 & ------- \\
(ESPM) & & \\
Totales &
\end{tabular}

Fuente: Elaboración propia.

\section{Resultados del trabajo realizado.}

\section{Problemas no resueltos:}

Destaca una alta incidencia de problemas no resueltos. El total de los 145 estudiantes del caso objeto de estudio, debían generar 1300 respuestas; por carreras: 150 de Lic. en Contabilidad y Finanzas, 260 en Lic. en Agronomía, 410 en Ing. Agrícola y 480 en Licenciatura en Pedagogía. Los resultados arrojaron por carreras: 127, 177, 326 y respuestas. En total se dejaron de resolver 190 problemas para un $23,2 \%$ de abstenciones. La tabla siguiente muestra estos resultados. 
Tabla 2. Resultados

\begin{tabular}{llllllll}
\hline Carr & $\begin{array}{l}\text { No } \\
\text { Est }\end{array}$ & $\begin{array}{l}\text { Cant. } \\
\text { Prob }\end{array}$ & $\begin{array}{l}\text { Tot Resp } \\
\text { Esperadas }\end{array}$ & $\begin{array}{l}\text { Tot Resp } \\
\text { Obtenid }\end{array}$ & $\begin{array}{l}\text { \% Resp } \\
\text { Obtenid }\end{array}$ & $\begin{array}{l}\text { Tot. } \\
\text { Absten }\end{array}$ & \% Absten \\
\hline Lic C F & 30 & 5 & 150 & 127 & 84,7 & 23 & 15,3 \\
Lic & 26 & 10 & 260 & 177 & 68,1 & 83 & 31,9 \\
Agr & & & & & & & \\
Ing. Ag & 41 & 10 & 410 & 326 & 79,5 & 84 & 20,5 \\
Lic Pe & 48 & 10 & 480 & 106 & 22,1 & 374 & 77,9 \\
Totales & 97 & --------- & 820 & 630 & 76,8 & 190 & 23,2 \\
\hline
\end{tabular}

Fuente: Elaboración propia.

\section{Estrategias y ejemplos significativos:}

A continuación, se relacionan y describen las estrategias utilizadas y se exponen algunos ejemplos más significativos. El orden en que aparecen descritas es creciente en cuanto al nivel de reflexión implícito que contienen.

3.1 Opera con los datos de forma irreflexiva (OCD): Estrategia en la cual se identifican los datos numéricos para operar con ellos irreflexivamente. Fonte (2003) lo asocia a la tendencia a la ejecución. Es la tercera estrategia más utilizada en la investigación.

\section{Ejemplo.}

Caso del Problema C. (Lic. en Cont. y Fin.): Multiplica 5500 por 4,40 obteniendo erróneamente 2 420. Su respuesta literal es: "En cada caja hay 2420 naranjas". Además, se muestra incapaz de explicar la lógica de su proceder.

3.2 Adivina la respuesta (AR): Consiste en emitir una respuesta sin justificación y sin un verdadero proceso de análisis de las condiciones del problema. Es la más utilizada en el estudio, manifestándose en el 36\% de las respuestas.

Ejemplo: (Lic. En Educac.): Escribe solo la respuesta literal: Están aprobados en ambas asignaturas 15 estudiantes. Es incapaz de explicar cómo obtuvo el resultado.

3.3 Mira los números y te dirán qué operaciones debes realizar (MLN): Consiste en asumir criterios acerca de la existencia de ciertas relaciones de carácter externo (pues no tienen que ver con sus significados) entre las operaciones aritméticas y los "tipos" de números con que operan. Fue utilizada solo en 5 casos.

\section{Ejemplo.}

Caso del problema F. (Licenciatura en Agronomía): El estudiante suma las velocidades de los dos primeros móviles $40+30=70$ y al resultado de la suma resta la velocidad del tercero 
$70-60=10$. Su respuesta es: "la menor distancia entre las dos ciudades es de $10 \mathrm{Km}$ ”. Al tratar de explicar la razón de su proceder, su argumentación incoherente pone el énfasis en el tipo de números de los datos: enteros de dos cifras (grandes) que pueden ser sumados y restados de acuerdo al texto.

3.4 Busca las palabras claves y ellas te dicen qué hacer, o sea, qué operación utilizar (PCLAV): Estrategia que se caracteriza por asociar el significado de las operaciones a ciertas palabras "claves" utilizadas en el propio proceso de enseñanza aprendizaje al trabajar con problemas. Fue utilizada en cinco de los diez problemas del test propuesto, manifestándose en 38 respuestas.

\section{Ejemplo.}

Caso del problema H (Ingeniería Agrícola):

Respuesta: Para mí, las dos recorren la pista al mismo tiempo, porque corren y caminan a la "misma velocidad". En la entrevista se evidencia que la palabra (frase) clave es: "la misma velocidad".

3.5 Procedimiento rutinario estudiado anteriormente (PREA). Estrategia asociada a dos creencias anteriormente aisladas por Fonte (2003). 1) "Los problemas se resuelven aplicando lo último estudiado en clase”; 2) "existen distintos tipos de problemas y para cada tipo un procedimiento de solución, para resolverlos solo hay que identificar el procedimiento correspondiente". Fue utilizada solo en 30 casos del total de respuestas manifestándose en ocho problemas.

\section{Ejemplo.}

Caso del problema D (Lic. En Agronomía).

El estudiante traza el diagrama que comenzó a utilizar para resolver situaciones tipo de 12mo grado.

Figura 1. Diagrama

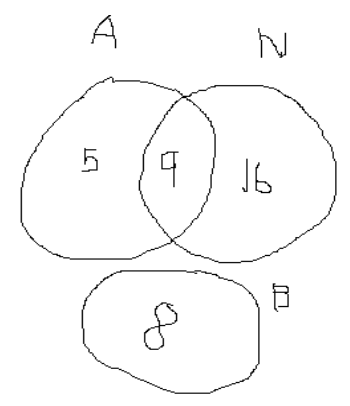

Datos: A (14); N (25); B (8)

Respuesta 1: Compiten solo en natación 16 y en atletismo 5 .

Respuesta 2: En la escuela hay un total de 29 alumnos.

Fuente: Elaboración propia. 
El estudiante asimiló el procedimiento para construir este tipo de diagrama. Sin embargo, no es capaz de determinar el total de estudiantes participantes de la carrera.

Procedimiento rutinario asociado a indicador textual (PRAIT): Consiste en identificar en el texto ciertos indicadores asociados a la clase de problemas en que se usa un determinado procedimiento, por ejemplos: \%, parte de un total $\left(\frac{m}{n}\right)$, etc. Se localiza en este estudio solo en los problemas $\mathrm{F}$ y $\mathrm{G}$.

3.6 Procedimiento rutinario con modelo algebraico (PRMA). Consiste en declarar variables cuyos significados no responden a las magnitudes y relaciones del texto. A partir de ellas se plantea y se trata de resolver una ecuación o un sistema de ecuaciones. Aparece en 24 procesos de solución asociada a la creencia de que para resolver problemas la vía más adecuada y efectiva es la de declarar variables.

\section{Ejemplo.}

Caso del problema B. (Lic. En Contabilidad y Finanzas).

Plantea lo siguiente: Alejandro: x; Emma: y. (Declaración de variables).

A continuación, plantea y trata de resolver el siguiente sistema de ecuaciones:

Figura 2. Sistema de ecuaciones.
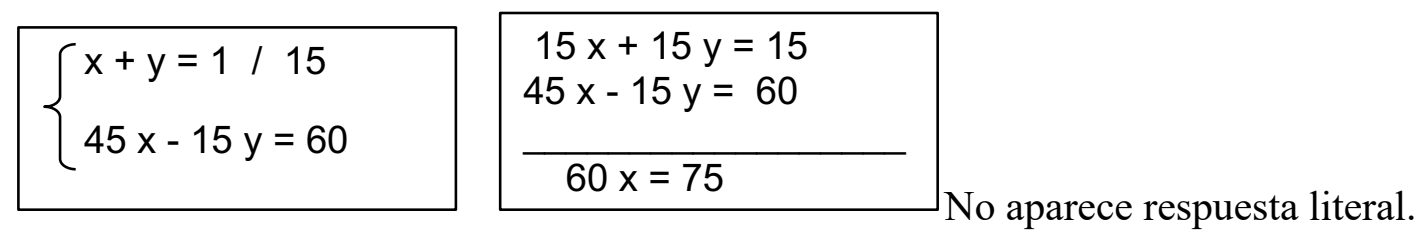

Fuente: Elaboración propia.

3.7 Tanteo inteligente (TANT). Procedimiento reflexivo que consiste en buscar la solución probando reiteradamente hasta encontrar la solución (búsqueda mediante ensayo-error). Fue utilizada una sola vez por un estudiante de Lic. en Contabilidad y Finanzas.

\section{Ejemplo.}

Caso único del problema F (Lic. en Contabilidad y Finanzas).

Plantea literalmente: La menor distancia que puede existir entre ambas ciudades es de $120 \mathrm{~km}$, ya que, si el carro que va a $40 \mathrm{~km} / \mathrm{h}$ se demora 3 horas exactas, obtendría un total de 120 $\mathrm{km} / \mathrm{h}$; si el carro que va a $30 \mathrm{~km} / \mathrm{h}$ demora 4 horas exactas obtendría $120 \mathrm{~km} / \mathrm{h}$ también y si el auto de $60 \mathrm{~km} / \mathrm{h}$ tarda 2 horas exactas en completar el recorrido también obtendría 120 $\mathrm{km} / \mathrm{h}$. 
Basa su razonamiento en que probando con otros números la distancia recorrida por los tres móviles le da numéricamente igual, pero siempre mayor que 120.

3.9. Usar números cómodos o razonables (UNC). Consiste en identificar, mediante adivinación o por estimados basados en aspectos no relevantes de la situación descrita, una combinación entre las magnitudes y luego probar que cumple las condiciones del problema. Fue utilizada en 7 procesos de solución:

3.10 Conteo directo sobre un modelo previa modelación (CDSM). Consiste en seleccionar de la realidad o describir (por lo general gráficamente) objetos o fenómenos que permiten modelar la situación dada despejándola de los elementos del texto que se consideran innecesarios, a la vez que se resaltan los que, a su juicio, resultan relevantes para el proceso de solución. En este estudio se aplicó en 39 casos.

\section{Ejemplo.}

Caso del problema I (Ing. Agrícola).

Figura 3. Caso del problema

$$
11+10+9+8+7+6+5+4+3+2+1
$$

Respuesta: Asistieron 12 estudiantes.

En la entrevista, el estudiante plantea que fue contando cada una de las rayitas que unen a los participantes equivalentes a los apretones de mano.

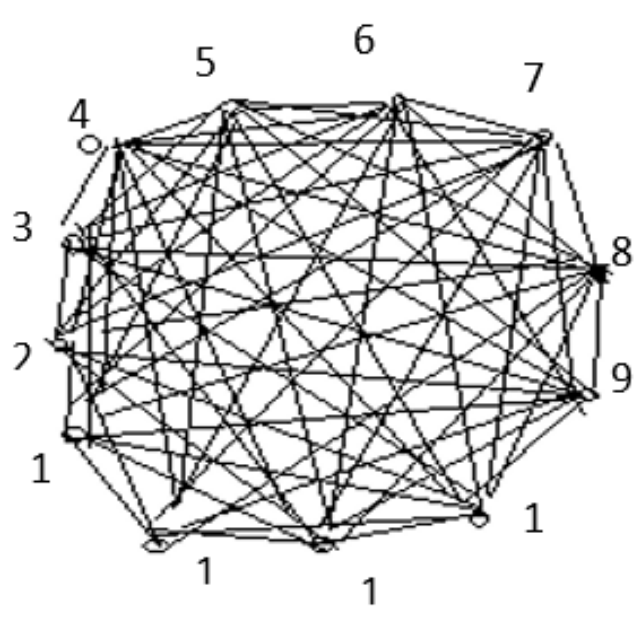

Fuente: Elaboración propia. 
3.11 Identificar el significado de las operaciones en el texto (ISOT). Consiste en analizar la situación reflejada en el problema e identificar y resolver las operaciones cuyos significados responden a la situación descrita. Utilizada en 181 respuestas, es la segunda estrategia más utilizada.

\subsection{Identifica información relevante y realiza inferencias deductivas e inductivas (IIR).}

Es una estrategia de alto nivel reflexivo, citada por Fonte (2003), no aislada en esa ocasión. Utilizada aquí por los estudiantes de las tres carreras, se genera la hipótesis de que esta es una estrategia que adquieren los estudiantes en un mayor nivel de escolaridad (superior en este caso).

Consiste en delimitar los elementos que conforman las premisas de la situación problémica e inferir la solución mediante procesos de análisis que van desde lo particular a leyes generales, o de desde estas a situaciones particulares.

\section{Ejemplo.}

Caso correspondiente al problema I (Lic. en Agronomía)

Figura 4. Caso del problema

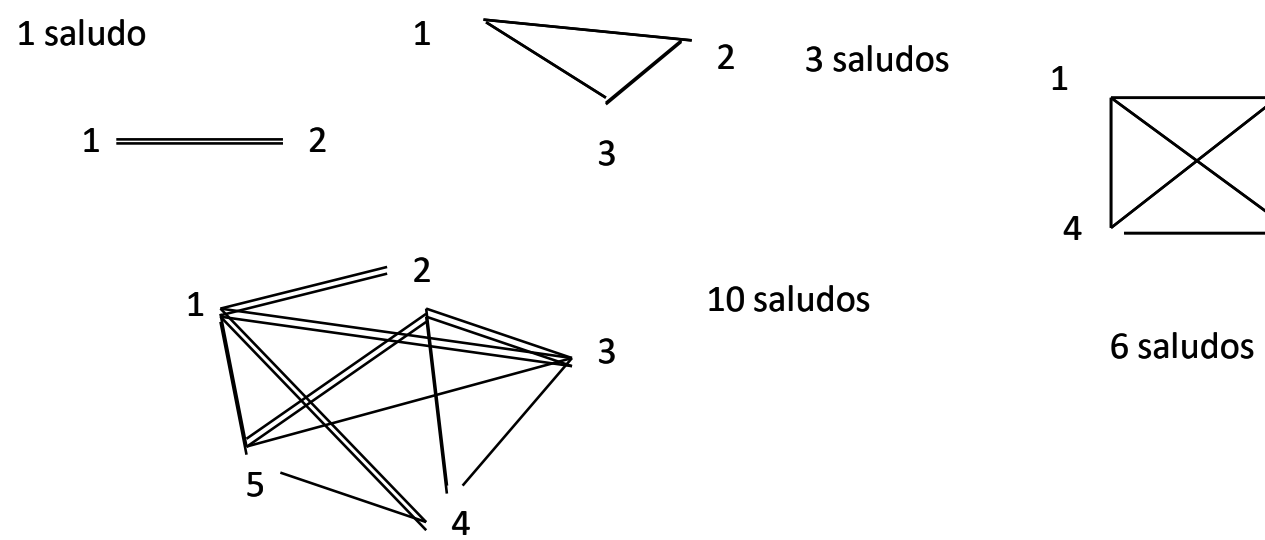

Fuente: Elaboración propia.

Dos estudiantes: 1 saludo; Tres estudiantes: tres saludos $2+1=3$; Cuatro estudiantes: 6 saludos $3+2+1=6$; Cinco estudiantes: 10 saludos $4+3+2+1=10$; Seis estudiantes: 15 saludos $5+4+3+2+1=15$; Siete estudiantes: 21 saludos $6+5+4+3+2+1=21$; Ocho estudiantes: 28 saludos $7+21=28$; Nueve estudiantes: 36 saludos $8+28=36$; Diez estudiantes: 45 saludos $9+36=45$; Once estudiantes: 55 saludos $10+45=55$; Doce estudiantes: 66 saludos $11+55=66$ 
En la entrevista el estudiante afirma que se dio cuenta que en sumas sucesivas se debe añadir el número de personas menos uno, ya que la persona no se saluda a sí misma.

\section{Aportes de la investigación al diagnóstico integral de los estudiantes.}

Este aspecto alcanza especial relevancia entre los resultados alcanzados; pues, conocer las capacidades de pensamiento lógico de los estudiantes trasciende los marcos de la asignatura Matemática. Mediante el estudio diagnóstico se ha obtenido información fidedigna acerca de las formas de pensamiento de cada uno de los estudiantes, sin son reflexivas o irreflexivas. La técnica del estudio de casos se constituye en un instrumento para realizar este tipo de diagnóstico.

\section{Creencias aisladas en el estudio.}

Se manifestaron con fuerza las dos siguientes:

1. Los problemas siempre tienen que contener datos numéricos, de manera que, para resolverlos, se puedan realizar operaciones de cálculo.

2. Para resolver cualquier tipo de problemas es necesario realizar los pasos siguientes:

1ro. Declaración de variables,

2do. Planteo de ecuaciones,

3ro. Resolver el sistema de ecuaciones,

4to. Dar la respuesta.

Ambas creencias tienen su origen en la denominada "tendencia a la ejecución", la cual induce al estudiante a resolver operaciones de cálculo o a la declaración de variables para plantear y resolver ecuaciones.

\section{Conclusiones.}

- Las estrategias de resolución de problemas que utilizan los estudiantes de los primeros años de las carreras Lic. en Contabilidad y Finanzas, Lic. en Agronomía e Ingeniería Agrícola, así como los de Lic. En Pedagogía de la ESPM, heredadas de los niveles de enseñanza precedentes, en su mayoría mantienen la condición de ser irreflexivas, y pueden constituirse en un freno para el proceso de enseñanza aprendizaje de las Matemáticas Superiores de estos estudiantes.

- Estas estrategias tienen su origen en el trabajo con problemas rutinarios en detrimento de la solución de verdaderos problemas, así como el pobre tratamiento de este tema 
en el caso de los estudiantes angolanos, para el entrenamiento y desarrollo del pensamiento lógico.

- Las creencias que mantienen los estudiantes al entrar a la educación superior constituyen limitantes para sus posibilidades de éxito en la solución de verdaderos problemas, lo cual puede influir también negativamente en el proceso de enseñanza aprendizaje de las Matemáticas Superiores.

- El estudio realizado deriva la hipótesis de la existencia de correlaciones entre los resultados académicos de los estudiantes de las carreras involucradas en el estudio y el tipo de estrategias que utilizan en la solución de problemas. Del carácter irreflexivo de las estrategias se presupone la obtención de malos resultados académicos en las Matemáticas Superiores.

- La metodología de los estudios de casos se constituye en un valioso instrumento con basamento científico el cual permite diagnosticar las estrategias y creencias en resolución de problemas de los estudiantes del primer año de las carreras.

\section{Recomendaciones.}

Los resultados obtenidos en esta investigación demuestran que, previo al comienzo del estudio de las Matemáticas Superiores, deben ser conocidas las estrategias y creencias que en resolución de problemas mantienen los estudiantes. Es por eso que se recomienda:

- Aplicar un diagnóstico individualmente inicial a los estudiantes de nuevo ingreso para identificar cuáles son las estrategias y creencias que poseen en resolución de problemas.

- Establecer un tratamiento diferenciado para eliminar las estrategias irreflexivas de solución de problemas y las creencias que frenan el desarrollo del pensamiento lógico de los estudiantes y fomentar el uso frecuente de estrategias heurísticas a partir de problematizar los contenidos de las Matemáticas Superiores.

- Confeccionar un programa para el tratamiento de la resolución de problemas e incorporarlo al currículo de la ESPM de los estudiantes de las especialidades pedagógicas de Matemática, Física y Computación.

- Divulgar los resultados de esta investigación como fuente de información a los docentes de las Matemáticas Superiores y de otras asignaturas en las cuales se requiera el uso de un relativo nivel de pensamiento lógico como vía para el aprendizaje. 
- Dar continuidad a esta investigación como vía para comprobar o descartar las hipótesis generadas en este trabajo.

\section{Referencias bibliográficas.}

Almeida B, A. Didáctica de la resolución de problemas matemáticos en la escuela media. Promet. Editorial Academia. La Habana 1999.

Álvarez, C. Hacia una escuela de excelencia. Editorial Academia. La Habana 1996.

Beyer, W. (2000). La resolución de problemas en la Primera Etapa de la Educación Básica y su implementación en el aula. Caracas Venezuela

Campistrous, L y Rizo, C (1999). Didáctica y Resolución de Problemas. ICCP, La Habana

Cárdenas, J y otros. (2013) Resolución de problemas de matemáticas y eva-luación: aspectos afectivos y cognitivos. En grupo de investigación DE-PROFE (ed.), Las emociones en la enseñanza y el aprendizaje de las cien-cias y las Matemáticas.

Chamoso, J. y otros. (2012). La reflexión como elemento de formación docente en matemáticas: análisis e instrumentos. Cuadernos de Investigación y Formación en Educación Matemática.

Coletivo de Autores. A Língua Portuguesa na Resolução de Problemas Ma-temáticos. http:Iiwww.repositório.ipsantarem.pt

Colectivo de autores ISPEJV (2000). Metodología de la Enseñanza de la Matemática. Tomo I y Tomo II. Editorial Pueblo y Educación.

Fonte, A. (2003). Estrategias que utilizan los alumnos de Secundaria Básica para resolver problemas. Un estudio de casos. La Habana.

Hernández A. J.Cómo estás en Matemáticas? Ejercicios complementarios de Ma-temática, para la profundización en la enseñanza preuniversitaria. Editorial Pueblo y Educación. 2001.

Labarrere, A. (1998). Cómo enseñar a los alumnos de primaria a resolver problemas. Editorial Pueblo y Educación. Ciudad de La Habana.

Padilla, E. (2014). Resolución de problemas en Matemática y su didáctica en el contexto de los nuevos programas. Quepos, Puntarenas, Costa Rica

Poggioli, (2000). Estrategias de solución de problemas. Enseñando a aprender. http:Iiwww.fpolar.org.veIpoggioilpoggio05.htm. 


\section{Para citar el artículo indexado}

Fonte Águila, A. A., \& Varela Nualles, M. (2020). Estrategias de resolución de problemas y creencias de estudiantes universitarios (estudio de casos). AlfaPublicaciones, 1(3), 6-19. https://doi.org/10.33262/ap.v1i3.5



El artículo que se publica es de exclusiva responsabilidad de los autores y no necesariamente reflejan el pensamiento de la Revista Alpha Publicaciones.

El artículo queda en propiedad de la revista y, por tanto, su publicación parcial y/o total en otro medio tiene que ser autorizado por el director de la Revista Alpha Publicaciones.
